IGC-09/6-2

\title{
Effective Constraints for Relativistic Quantum Systems
}

\author{
Martin Bojowald and Artur Tsobanjant \\ Institute for Gravitation and the Cosmos, The Pennsylvania State University, \\ 104 Davey Lab, University Park, PA 16802, USA
}

\begin{abstract}
Determining the physical Hilbert space is often considered the most difficult but crucial part of completing the quantization of a constrained system. In such a situation it can be more economical to use effective constraint methods, which are extended here to relativistic systems as they arise for instance in quantum cosmology. By side-stepping explicit constructions of states, such tools allow one to arrive much more feasibly at results for physical observables at least in semiclassical regimes. Several questions discussed recently regarding effective equations and state properties in quantum cosmology, including the spreading of states and quantum back-reaction, are addressed by the examples studied here.
\end{abstract}

\section{Introduction}

One of the key issues in quantizations of fundamental theories, which due to their covariance properties are systems with gauge freedom generated by constraints, is the determination of physical observables. They must satisfy the constraint equations and be invariant under gauge transformations. For canonical quantum theories, solving constraints is traditionally done at the state level: one constructs a physical Hilbert space of states annihilated by the constraint operator(s) and equipped with an invariant inner product. Explicit constructions can be done in some special cases by different methods.

Since explicit derivations are possible only in specific cases, it is not always clear whether the results are generic or mere artefacts of the simple models used. It is therefore important to have approximate methods for a wider range of cases, or at least to be able to perturb around known solvable ones while still ensuring that the constraints are solved and the observables are gauge invariant. It turns out that such perturbation schemes are most feasible if one deals with the observables directly, such as expectation values, sidestepping the computation and physical normalization of states. This procedure gives rise to canonical effective equations and constraints [1, 2].

A procedure for effective constraints has been formulated in [2] and applied to parameterized non-relativistic systems with a constraint $p_{t}+H=0$ where $p_{t}$ is the momentum

*e-mail address: bojowald@gravity.psu.edu

$\dagger$ e-mail address: axt236@psu.edu 
of time and $H$ the Hamiltonian. (The concepts and results are reviewed briefly below.) It was shown that the physical observables in suitable regimes, including semiclassical ones, can be determined without making use of a physical inner product but instead through implementing reality conditions for quantum variables such as fluctuations, correlations and higher moments. For applications of these methods to quantum gravity and cosmology one has to extend them to relativistic systems, offering one additional subtlety: constraints would now be of the form $p_{t}^{2}-H^{2}=0$, requiring one to take a square root and to make sign choices. Mathematically, for instance, the question is how to precisely define $\sqrt{H^{2}}=|H|$ at the operator level. This may not be obvious if the operator $\mathbf{H}$ has a complicated spectrum or is not positive definite. Physically, one must decide how to treat and separate positive and negative frequency solutions corresponding to the two solutions $p_{t}= \pm|H|$. (See e.g. [3, 4] for discussions of relativistic systems.)

For a time-independent Hamiltonian, it turns out that one can, at least for semiclassical purposes, simply use $p_{t}= \pm\langle\mathbf{H}\rangle$ as the effective Hamiltonian [5, 6] without absolute values, even if $\mathbf{H}$ is not positive definite. One only has to ensure that the initial values used in the effective equations of motion correspond to an initial state supported on a part of the spectrum of $\mathbf{H}$ with a definite sign. On such a state and with a self-adjoint $\mathbf{H},\langle|\mathbf{H}|\rangle=\langle\mathbf{H}\rangle$ if the state is supported only on the positive part of the spectrum of $\mathbf{H}$, and $\langle|\mathbf{H}|\rangle=-\langle\mathbf{H}\rangle$ if the state is supported only on the negative part. Since the Hamiltonian is preserved, these statements will hold true throughout the whole evolution and there is no need for an absolute value in the effective Hamiltonian. This fact has been made use of in several recent derivations of effective equations in quantum cosmology, where the relevant versions of $\mathbf{H}$ are not positive definite [7, 8].

In those models, deparameterization was performed using $t=\phi$ as an internal time from a free, massless scalar $\phi$. The same types of models also allow the construction of a physical Hilbert space at the state level [9, 10, with the results in agreement with those obtained from effective equations. Most interesting from the cosmological perspective is, however, a system where the scalar $\phi$ has a non-trivial potential or at least a mass term. This has two immediate implications: in general, one can no longer deparameterize globally since the solutions for the scalar would not be monotonic in the time coordinates, and the Hamiltonian would no longer be (internal) time independent. Positivity can no longer be ensured just by an initial condition, and using $p_{\phi}= \pm\langle\mathbf{H}(\phi)\rangle$ as an effective Hamiltonian without an absolute value may then seem questionable. Explicitly using an absolute value, on the other hand, would make a derivation of the effective equations more complicated. At this stage, a direct treatment of effective constraints for relativistic systems without deparameterization becomes relevant. This is what we present in the current paper.

We will consider in detail models of relativistic particles and properties of observables in their quantum theories. In the massive case, for instance, we are dealing with the quantization and implementation of a constraint $C=p_{t}^{2}-p^{2}-m^{2}$. For physical states, the expectation value

$$
\langle\mathbf{C}\rangle=p_{t}^{2}+\left(\Delta p_{t}\right)^{2}-p^{2}-(\Delta p)^{2}-m^{2}
$$

must vanish and thus represents a quantum constraint. (We will notationally identify 
classical degrees of freedom with the expectation values to simplify the notation and to show the relation between classical terms and quantum corrections. Thus, $E=\langle\mathbf{E}\rangle$ and $(\Delta E)^{2}=\left\langle(\mathbf{E}-\langle\mathbf{E}\rangle)^{2}\right\rangle=\left\langle\mathbf{E}^{2}\right\rangle-E^{2}$.) As we will see in more detail below, there are additional independent constraints since expressions such as $\langle\mathbf{q C}\rangle$ must also vanish for physical states. Allowing all possible factors to the left of $\mathbf{C}$, this presents a constrained system of infinitely many constraints for infinitely many quantum variables given by the moments of a state. The combined system of all constraints must be solved to find observable results, which is feasible in semiclassical regimes where only a finite set of moments suffices to characterize a state approximately. The same kind of approximation also allows one to include potentials within the constraint, which may be explicitly time-dependent. We will exploit this to justify the procedures used in quantum cosmology for deparameterized systems with timedependent potentials, as developed in [11, 12, 13].

Another question of interest is that of the spreading of states and quantum backreaction of fluctuations and higher moments on the expectation values. If we compare the effective constraint $\langle\mathbf{C}\rangle$ written above, which contains only the second order moments in addition to the expectation values, with the effective Hamiltonian of the corresponding deparameterized system,

$$
\begin{aligned}
\langle\mathbf{H}\rangle & =\left\langle\sqrt{\mathbf{p}^{2}+m^{2}}\right\rangle=\left\langle\sqrt{(p+(\mathbf{p}-p))^{2}+m^{2}}=\sqrt{p^{2}+m^{2}}+\sum_{n=2}^{\infty} \frac{1}{n !} \frac{\partial^{n} \sqrt{p^{2}+m^{2}}}{\partial p^{n}}\left\langle(\mathbf{p}-p)^{n}\right\rangle\right. \\
& =\sqrt{p^{2}+m^{2}}+\frac{m^{2}(\Delta p)^{2}}{\left(p^{2}+m^{2}\right)^{3 / 2}}-3 m^{2} \frac{m^{2}-4 p^{2}}{\left(p^{2}+m^{2}\right)^{7 / 2}}\left\langle(\mathbf{p}-p)^{3}\right\rangle+\cdots
\end{aligned}
$$

with a whole formal Taylor series that includes higher moments, different coupling terms between the expectation values and the moments seem to be implied. Then, back-reaction might seem different in these two treatments, apparently making them incompatible. By our specific constructions in this paper we will reconcile these apparent disagreements, and provide an illustration by numerical solutions in a specific example (App. B). This is also important for quantum cosmology, where quantum back-reaction is crucial to the understanding of how a quantum state evolves toward and possibly through the big bang and how much of the pre-big bang state can be reconstructed [14, 15].

\section{Effective constraints}

In a canonical effective description, the dynamics of a quantum system with $n$ degrees of freedom is formulated in terms of the expectation values, i.e. the evaluations of a state functional in the elements of an algebra $\mathscr{A}$ generated by $2 n$ basic operators $\mathbf{q}_{i}$ and $\mathbf{p}_{i}$, $i=1, \ldots, n$. This whole set of infinitely many variables can be conveniently split into the $2 n$ expectation values of the basic operators, such as $q_{i}=\left\langle\mathbf{q}_{i}\right\rangle$ and $p_{i}=\left\langle\mathbf{p}_{i}\right\rangle, i=1, \ldots, n$, together with the infinitely many moments of the form

$$
G^{\left\{a_{j}\right\},\left\{b_{j}\right\}}=\left\langle\prod_{i=1}^{n}\left(\mathbf{q}_{i}-\left\langle\mathbf{q}_{i}\right\rangle\right)^{a_{i}}\left(\mathbf{p}_{i}-\left\langle\mathbf{p}_{i}\right\rangle\right)^{b_{i}}\right\rangle_{\text {Weyl }}
$$


where the subscript "Weyl" denotes the totally symmetric ordering of all factors involved. Between all these variables a Poisson bracket is defined following from the algebra of commutation relations:

$$
\{\langle\mathbf{A}\rangle,\langle\mathbf{B}\rangle\}=\frac{\langle[\mathbf{A}, \mathbf{B}]\rangle}{i \hbar}
$$

extended using linearity and the Leibniz rule.

At second order, $\sum_{i=1}^{n}\left(a_{i}+b_{i}\right)=2$, this set of moments includes all fluctuations and covariances. In semiclassical regimes, moments fall off at least as $\hbar^{\frac{1}{2} \sum_{i=1}^{n}\left(a_{i}+b_{i}\right)}$ such that only low orders need to be considered for the first approximation to quantum effects. Below we will employ the notation $(\Delta a)^{2}=\left\langle(\mathbf{a}-a)^{2}\right\rangle$ for fluctuations and $\Delta(a b)=$ $\langle(\mathbf{a}-a)(\mathbf{b}-b)\rangle_{\text {Weyl }}=\frac{1}{2}\langle(\mathbf{a}-a)(\mathbf{b}-b)+(\mathbf{b}-b)(\mathbf{a}-a)\rangle$ for covariances, which may make second order equations easier to interpret.

Any operator $\mathbf{C}=C\left(\mathbf{q}_{i}, \mathbf{p}_{j}\right)$ gives rise to a function on the space of states, which can be expressed in terms of the moments by Taylor expanding

$$
\left\langle C\left(\mathbf{q}_{i}, \mathbf{p}_{j}\right)\right\rangle=\left\langle C\left(q_{i}+\left(\mathbf{q}_{i}-q_{i}\right), p_{j}+\left(\mathbf{p}_{j}-p_{j}\right)\right)\right\rangle
$$

in $\mathbf{q}_{i}-q_{i}$ and $\mathbf{p}_{j}-p_{j}$ as in (11). If $C\left(\mathbf{q}_{i}, \mathbf{p}_{j}\right)$ is a constraint operator, (3) must vanish on physical states and thus is a constraint on the quantum phase space.

A single constraint on the phase space removes one pair of variables, but not the whole tower of moments associated with it in the quantum phase space. For a complete reduction one has to make use of additional constraints, provided by the set of phase space functions $\left\langle f\left(\mathbf{q}_{i}, \mathbf{p}_{i}\right) C\left(\mathbf{q}_{j}, \mathbf{p}_{k}\right)\right\rangle$ which must also vanish on physical states. These functions are in general independent from the quantum constraint $\left\langle C\left(\mathbf{q}_{i}, \mathbf{p}_{j}\right)\right\rangle$ as functions of expectation values and moments. As shown in [2], this set of constraints remains first class. (Note that we do not order the products of the operators in the constraints symmetrically to ensure that the constraint operator acts directly on the state. As a result, in some cases one has to deal with complex-valued constraints requiring reality conditions for physical observables. This has been discussed in [2] and will also be seen in more detail in the examples below.)

Given such a system of constraints on the quantum phase space, one can proceed in the classical way and find the reduced quantum phase space of observables or solve the constraints and fix the gauge. At this stage, it is convenient (but not required) to decide which internal time variables $\left(t, p_{t}\right)$ among the $\left(q_{i}, p_{j}\right)$ should be used. Since a quantization of the corresponding deparameterized system, if it exists, would not give rise to any moment involving an operator of $t$ or $p_{t}$, solving the constraints must remove all moments including at least one factor of $\mathbf{t}$ or $\mathbf{p}_{t}$ from the original quantum phase space. That this indeed happens was verified to second order of the parameterized non-relativistic particle in [2]. In that case, there was a single linear term $p_{t}$ in the constraint, such that all $p_{t}$-moments $\left(\Delta p_{t}\right)^{2}, \Delta\left(p_{t} t\right), \Delta\left(p_{t} q\right)$ and $\Delta\left(p_{t} p\right)$ can be removed by solving the system of constraints, to 
second order:

$$
\begin{aligned}
\langle(\mathbf{q}-q) \mathbf{C}\rangle & =\Delta\left(q p_{t}\right)+\frac{p}{M} \frac{i \hbar}{2}+\frac{p}{M} \Delta(q p)=0 \\
\langle(\mathbf{t}-t) \mathbf{C}\rangle & =\frac{p}{M} \Delta(p t)+\Delta\left(t p_{t}\right)+\frac{i \hbar}{2}=0 \\
\left\langle\left(\mathbf{p}_{t}-p_{t}\right) \mathbf{C}\right\rangle & =\left(\Delta p_{t}\right)^{2}+\frac{p}{M} \Delta\left(p p_{t}\right)=0 \\
\langle(\mathbf{p}-p) \mathbf{C}\rangle & =\Delta\left(p p_{t}\right)+\frac{p}{M}(\Delta p)^{2}=0 .
\end{aligned}
$$

This leaves the moments $(\Delta t)^{2}, \Delta(t q)$ and $(\Delta t p)$, which are removed by factoring out the gauge flow, or simply by setting them to zero as a well-defined set of gauge-fixing conditions. (Note that a smaller number of gauge fixing conditions than constraints is required because the second order moments satisfy a Poisson algebra which is degenerate from the symplectic point of view; see [16] for some notions of constrained systems in the non-symplectic case. Additionally, setting the fluctuation $(\Delta t)^{2}$ to zero is consistent with the generalized uncertainty relation

$$
(\Delta t)^{2}\left(\Delta p_{t}\right)^{2}-\Delta\left(t p_{t}\right)^{2} \geq \frac{\hbar^{2}}{4}
$$

since $t-p_{t}$-correlations $\Delta\left(t p_{t}\right)=-i \hbar / 2$ are required by the constraints, especially $\langle(\mathbf{t}-$ $t) \mathbf{C}\rangle=0$ with the gauge fixing condition $\Delta(p t)=0$, to be imaginary and of just the right size to saturate the uncertainty relation.) After solving the constraints and fixing the gauge, observable moments are recovered on which physical reality conditions can be imposed.

\section{Free relativistic particle}

Classically a free relativistic particle is described by a single constraint

$$
C=p_{t}^{2}-p^{2}-m^{2}
$$

on the phase space coordinatized by two canonical pairs $t, p_{t}$ and $q, p 3$ For the quantum version we consider the unital associative algebra generated by four basic elements $\mathbf{t}, \mathbf{p}_{t}, \mathbf{q}, \mathbf{p}$ subject to the canonical commutator relations. That is, $\mathscr{A}$ consists of (countable) sums of polynomials of the form $\mathbf{t}^{i} \mathbf{p}_{t}^{j} \mathbf{q}^{k} \mathbf{p}^{l}$; terms with a different ordering may be expressed using the commutation relations

$$
\left[\mathbf{t}, \mathbf{p}_{t}\right]=i \hbar \mathbb{1} \quad, \quad[\mathbf{q}, \mathbf{p}]=i \hbar \mathbb{1}
$$

There is no product-ordering ambiguity in the case of the above constraint and it is naturally identified with an element $\mathbf{C}=\mathbf{p}_{t}^{2}-\mathbf{p}^{2}-m^{2} \mathbb{1}$ of $\mathscr{A}$. As the equivalent of Dirac's

\footnotetext{
${ }^{3}$ We assume the units have been chosen so that both length and momentum have the units of the square root of action (e.g. geometrized units).
} 
condition $\mathbf{C} \psi=0$ we demand that the constraint has a vanishing right action on the states, which in our case are complex linear functions $\alpha: \mathscr{A} \rightarrow \mathbb{C}$, this implies that $\alpha(\mathbf{a C})=0, \forall \mathbf{a} \in \mathscr{A}$; henceforth we drop explicit reference to $\alpha$ and write this condition as $\langle\mathbf{a C}\rangle=0$ for the expectation value in a physical state $\alpha$. In order to impose all these conditions systematically we take the previously mentioned basis in $\mathscr{A}$ and impose the constraint via an infinite (but countable) set of conditions

$$
\left\langle\mathbf{t}^{i} \mathbf{p}_{t}^{j} \mathbf{q}^{k} \mathbf{p}^{l} \mathbf{C}\right\rangle=0
$$

We reduce the above infinite system of equations using the same method that was previously employed for a Newtonian particle [2] - a semiclassical expansion based on the hierarchy

$$
\left\langle(\mathbf{t}-\langle\mathbf{t}\rangle)^{i}\left(\mathbf{p}_{t}-\left\langle\mathbf{p}_{t}\right\rangle\right)^{j}(\mathbf{q}-\langle\mathbf{q}\rangle)^{k}(\mathbf{p}-\langle\mathbf{p}\rangle)^{l}\right\rangle_{\mathrm{Weyl}} \propto \hbar^{\frac{1}{2}(i+j+k+l)}
$$

of moments.

\subsection{Constraints at second order}

In what follows, we assume a semiclassical state and drop the terms of order $\hbar^{\frac{3}{2}}$ and above, keeping the terms of order $\hbar$ and below. This will suffice to demonstrate the feasibility of our methods for relativistic systems. To this order our system is described by fourteen independent functions: four expectation values of the form $a=\langle\mathbf{a}\rangle$; four spreads of the form $(\Delta a)^{2}=\left\langle(\mathbf{a}-a)^{2}\right\rangle$ and six covariances of the form $\Delta(a b)=\langle(\mathbf{a}-a)(\mathbf{b}-b)\rangle_{\text {Weyl }}$. (Poisson brackets between all these variables are listed in App. A.) The infinite system of constraint functions is reduced to just five non-trivial conditions

$$
\begin{aligned}
C & =\langle\mathbf{C}\rangle=p_{t}^{2}-p^{2}-m^{2}+\left(\Delta p_{t}\right)^{2}-(\Delta p)^{2}=0 \\
C_{t} & =\langle(\mathbf{t}-\langle\mathbf{t}\rangle) \mathbf{C}\rangle=2 p_{t} \Delta\left(t p_{t}\right)+i \hbar p_{t}-2 p \Delta(t p)=0 \\
C_{p_{t}} & =\left\langle\left(\mathbf{p}_{t}-\left\langle\mathbf{p}_{t}\right\rangle\right) \mathbf{C}\right\rangle=2 p_{t}\left(\Delta p_{t}\right)^{2}-2 p \Delta\left(p_{t} p\right)=0 \\
C_{q} & =\langle(\mathbf{q}-\langle\mathbf{q}\rangle) \mathbf{C}\rangle=2 p_{t} \Delta\left(p_{t} q\right)-2 p \Delta(q p)-i \hbar p=0 \\
C_{p} & =\langle(\mathbf{p}-\langle\mathbf{p}\rangle) \mathbf{C}\rangle=2 p_{t} \Delta\left(p_{t} p\right)-2 p(\Delta p)^{2}=0 .
\end{aligned}
$$

Note that the semiclassical hierarchy of variables is critical to the above reduction in the number of constraint conditions. In particular, $C=0$ implies that $p_{t}^{2}-p^{2}-m^{2}=$ $(\Delta p)^{2}-\left(\Delta p_{t}\right)^{2}$, which in turn implies that the combination of the expectation values $C_{\text {Class }}:=p_{t}^{2}-p^{2}-m^{2}$ is of order $\hbar$ on the constraint surface. In other words, the classical constraint is satisfied to order $\hbar$. The terms of the form $(\Delta a)^{2} C_{\text {Class }}$ and $\Delta(a b) C_{\text {Class }}$ are then of order $\hbar^{2}$ and should be dropped in our present treatment. The complete infinite system of constraint functions is a closed Poisson algebra - or, in the language of classical constraint analysis, a first-class system [17]. In general, due to the nature of the above truncation one would expect the reduced system of constraints to remain closed only to order $\hbar$. In our case the Poisson algebra of the truncated set of constraint functionsdisplayed in Table 1 - is exactly closed with respect to the bracket. 
Table 1: Poisson algebra of constraints for a free particle. First terms in the bracket are labeled by rows, second terms are labeled by columns.

\begin{tabular}{|c||c|c|c|c|c|}
\hline & $C$ & $C_{t}$ & $C_{p_{t}}$ & $C_{q}$ & $C_{p}$ \\
\hline \hline$C$ & 0 & $-2 C_{p_{t}}$ & 0 & $-2 C_{p}$ & 0 \\
\hline$C_{t}$ & $2 C_{p_{t}}$ & 0 & $4 p_{t} C_{p_{t}}-2 p C_{p}$ & $2 p C_{t}+2 p_{t} C_{q}$ & $2 p_{t} C_{p}$ \\
\hline$C_{p_{t}}$ & 0 & $2 p C_{p}-4 p_{t} C_{p_{t}}$ & 0 & $2 p C_{p_{t}}$ & 0 \\
\hline$C_{q}$ & $2 p C_{p}$ & $-2 p C_{t}-2 p_{t} C_{q}$ & $-2 p C_{p_{t}}$ & 0 & $2 p_{t} C_{p_{t}}-4 p C_{p}$ \\
\hline$C_{p}$ & 0 & $-2 p_{t} C_{p}$ & 0 & $4 p C_{p}-2 p_{t} C_{p_{t}}$ & 0 \\
\hline
\end{tabular}

To solve the constraint system we eliminate five variables using the five conditions from (5). Specifically, we eliminate the five quantum variables associated with $\mathbf{p}_{t}$, having in mind that $t$ will be chosen as time in a deparameterized treatment. We start by noting that $p_{t} C_{p_{t}}=0$ gives

$$
0=p_{t}^{2}\left(\Delta p_{t}\right)^{2}-p p_{t} \Delta\left(p_{t} p\right)
$$

However $C_{p}=0$ implies

$$
p_{t} \Delta\left(p_{t} p\right)=p(\Delta p)^{2}
$$

and substituted in (6) gives

$$
0=p_{t}^{2}\left(\Delta p_{t}\right)^{2}-p^{2}(\Delta p)^{2} .
$$

Finally, eliminating $p_{t}^{2}$ through $C=0$ we obtain a quadratic equation in $\left(\Delta p_{t}\right)^{2}$

$$
\left(\left(\Delta p_{t}\right)^{2}\right)^{2}-\left(\Delta p_{t}\right)^{2}\left(p^{2}+m^{2}+(\Delta p)^{2}\right)+p^{2}(\Delta p)^{2}=0
$$

with two solutions

$$
\left(\Delta p_{t}\right)^{2}=\frac{1}{2}\left(p^{2}+m^{2}+(\Delta p)^{2} \pm \sqrt{\left(p^{2}+m^{2}+(\Delta p)^{2}\right)^{2}-4 p^{2}(\Delta p)^{2}}\right) .
$$

In order to see whether either solution is compatible with the hierarchy assumed by the semiclassical approximation, we expand the solution to order $\hbar$. One finds

$$
\begin{aligned}
\left(\Delta p_{t}\right)^{2} & =\frac{1}{2}\left(p^{2}+m^{2}\right)\left(1+\frac{(\Delta p)^{2}}{p^{2}+m^{2}} \pm \sqrt{1+\frac{(\Delta p)^{2}\left(2 m^{2}-2 p^{2}+(\Delta p)^{2}\right)}{\left(p^{2}+m^{2}\right)^{2}}}\right) \\
& =\frac{1}{2}\left(p^{2}+m^{2}\right)\left(1 \pm 1+\frac{(\Delta p)^{2}}{\left(p^{2}+m^{2}\right)^{2}}\left(p^{2}+m^{2} \pm\left(m^{2}-p^{2}\right)\right)+O\left((\Delta p)^{4}\right)\right) .
\end{aligned}
$$

Looking at the solution with the "+" sign we see the following leading order behavior

$$
\left(\Delta p_{t}\right)^{2}=p^{2}+m^{2}+O(\hbar)
$$

which is inconsistent with the assumption that $\left(\Delta p_{t}\right)^{2}$ is of order $\hbar$. The solution with the "-" sign leads to

$$
\left(\Delta p_{t}\right)^{2}=\frac{p^{2}(\Delta p)^{2}}{p^{2}+m^{2}}+O\left((\Delta p)^{4}\right)
$$


which is of order $\hbar$ and therefore consistent with the semiclassical approximation. Substituting the latter solution back into the constraint conditions (5) we obtain two sets of solutions

$$
\begin{aligned}
p_{t} & = \pm E \\
\Delta\left(t p_{t}\right) & = \pm \frac{p}{E} \Delta(t p)-\frac{i \hbar}{2} \\
\left(\Delta p_{t}\right)^{2} & =p^{2}+m^{2}+(\Delta p)^{2}-E^{2} \\
\Delta\left(p_{t} q\right) & = \pm \frac{p}{E}\left(\Delta(q p)+\frac{i \hbar}{2}\right) \\
\Delta\left(p_{t} p\right) & = \pm \frac{p}{E}(\Delta p)^{2}
\end{aligned}
$$

where

$$
E=\frac{1}{\sqrt{2}}\left(p^{2}+m^{2}+(\Delta p)^{2}+\sqrt{\left(p^{2}+m^{2}+(\Delta p)^{2}\right)^{2}-4 p^{2}(\Delta p)^{2}}\right)^{\frac{1}{2}} .
$$

By rearranging the terms in the above expression one can easily verify that $E \geq 0$ when reality and positivity of the physical variables are imposed (see Section 3.2). One recovers the usual relativistic dispersion relation where energy equals $\sqrt{p^{2}+m^{2}}$ if one assumes a "momentum eigenstate", that is if one sets the spread in momentum $(\Delta p)^{2}=0$.

\subsection{Gauge freedom}

The truncated system of constraints (5) is equivalent (when consistency with the semiclassical approximation is evoked) to the restriction to two disjoint surfaces, each one corresponding to a choice of sign in (77). Each surface is described by an equivalent set of "linearized" constraints

$$
\begin{aligned}
& C_{1 \pm}=p_{t} \pm E \\
& C_{2 \pm}=\left(\Delta p_{t}\right)^{2}-p^{2}-m^{2}-(\Delta p)^{2}+E^{2} \\
& C_{3 \pm}=\Delta\left(p_{t} p\right) \pm \frac{p}{E}(\Delta p)^{2} \\
& C_{4 \pm}=\Delta\left(p_{t} q\right) \pm \frac{p}{E}\left(\Delta(q p)+\frac{i \hbar}{2}\right) \\
& C_{5 \pm}=\Delta\left(t p_{t}\right) \pm \frac{p}{E} \Delta(t p)+\frac{i \hbar}{2} .
\end{aligned}
$$

The above constraint conditions can be expressed as sums of the conditions in (5) and therefore form a first class system. Additionally, for the calculations to follow it is useful to note that $p_{t}, p,\left(\Delta p_{t}\right)^{2}, \Delta\left(p_{t} p\right)$ and $(\Delta p)^{2}$ are first class functions with respect to either set of constraints. This can ultimately be traced back to the fact that $\left[\mathbf{p}_{t}, \mathbf{C}\right]=0=[\mathbf{p}, \mathbf{C}]$. It follows that $E$, which is a function of $p$ and $(\Delta p)^{2}$ only, is also first class.

On the constraint surfaces the "linearized" constraints can be used to eliminate the five variables $p_{t},\left(\Delta p_{t}\right)^{2}, \Delta\left(t p_{t}\right), \Delta\left(p_{t} p\right), \Delta\left(p_{t} q\right)$. At this stage there remain four degrees 
of freedom associated with the algebra elements generated by $\mathbf{t}$. These will be treated as gauge parameters associated with the time evolution of the system. From this point of view, one has a four-parameter space to choose from when it comes to the evolution of the "physical variables" (i.e. those associated with the algebra generated by $\mathbf{q}$ and $\mathbf{p}$ alone). Viewing our system expanded to second order in quantum variables as a classical constrained system, the evolution on the "physical variables" $-q, p,(\Delta q)^{2}, \Delta(q p),(\Delta p)^{2}$ - may be generated by any constraint function of the form

$$
C_{\mathrm{Ham}}=\sum_{i} \mu_{i} C_{i \pm}
$$

where the multipliers $\mu_{i}$ are arbitrary functions of the physical variables. The presence of several constraints, all associated with the classical Hamiltonian, means that a priori there is no unique time parameter. Depending on the choice of gauge, any combination of $t$ with moments involving $\mathbf{t}$ can play the role of time.

At this stage we would like to restrict the gauge freedom down to a single parameter and to interpret the first class flow in the direction of $t$ as the dynamical evolution of the system. This may be accomplished by introducing three gauge choices

$$
\begin{aligned}
& \phi_{1}=(\Delta t)^{2}-f_{1}\left(q, p,(\Delta q)^{2}, \Delta(q p),(\Delta p)^{2}\right)=0 \\
& \phi_{2}=\Delta(t q)-f_{2}\left(q, p,(\Delta q)^{2}, \Delta(q p),(\Delta p)^{2}\right)=0 \\
& \phi_{3}=\Delta(t p)-f_{3}\left(q, p,(\Delta q)^{2}, \Delta(q p),(\Delta p)^{2}\right)=0
\end{aligned}
$$

with functions $f_{1}, f_{2}$ and $f_{3}$ to be determined. We define $C_{\text {Ham }}$ as the first class function (9) that remains after the gauge conditions have been introduced. It must therefore be a combination of the original constraint functions as in equation (9) that in addition satisfies

$$
\left\{C_{\text {Ham }}, \phi_{i}\right\} \approx 0, \quad i=1,2,3
$$

where the symbol ' $\approx$ ' denotes equality on the surface defined by imposing both the constraints and the gauge conditions. A simple set of such conditions that was also used in Ref. [2] to recover the deparameterized dynamics of a Newtonian particle is provided by

$$
\begin{aligned}
& \phi_{1}=(\Delta t)^{2}=0 \\
& \phi_{2}=\Delta(t q)=0 \\
& \phi_{3}=\Delta(t p)=0 .
\end{aligned}
$$

(Again, $(\Delta t)^{2}=0$ is consistent with the uncertainty relation since $\Delta\left(t p_{t}\right)=-i \hbar / 2$ from $C_{5 \pm}$.)

Let $\Sigma_{ \pm}$be the surfaces defined by simultaneously imposing the constraints $\left\{C_{i \pm}\right\}$ and the above gauge conditions. These are coordinatized by the physical variables $-q, p,(\Delta q)^{2}$, $\Delta(q p),(\Delta p)^{2}$ - and the one remaining gauge degree of freedom- $t$. It is straightforward to verify that the variables $(\Delta t)^{2}, \Delta(t q)$ and $\Delta(t p)$ generate a Poisson ideal of the algebra of physical and gauge variables (i.e. the variables that do not involve $\mathbf{p}_{t}$ ). That is, $\left\{\phi_{i}, X\right\}$ 
is a sum of gauge conditions with some coefficients. It follows that on $\Sigma_{ \pm}$the gauge-fixing conditions have a trivial Poisson algebra $\left\{\phi_{i}, \phi_{j}\right\} \approx 0$ and a vanishing Poisson bracket with the remaining free variables. It is not difficult to see that $C_{1 \pm}$ remains first class on $\Sigma_{ \pm}$. Since $E$ is a function of the "physical" variables only, $\left\{E, \phi_{i}\right\} \approx 0$ and so

$$
\left\{\phi_{i}, C_{1 \pm}\right\}=\left\{\phi_{i}, p_{t} \pm E\right\} \approx 0 .
$$

Furthermore, writing $C_{5 \pm}=\Delta\left(t p_{t}\right) \pm \phi_{3} p / E+$ const. one can quickly establish that $C_{5 \pm}$ also remains first class but has a vanishing Poisson flow on $\Sigma_{ \pm}$.

The remaining set of surface-defining conditions composed of $C_{2 \pm}, C_{3 \pm}, C_{4 \pm}$ and $\left\{\phi_{i}\right\}_{i=1,2,3}$ is second class for all admissible values of the physical variables. This can be seen by relabeling the conditions as $\chi_{1}=C_{2 \pm}, \chi_{2}=C_{3 \pm}, \chi_{3}=C_{4 \pm}, \chi_{4}=\phi_{1}, \chi_{5}=\phi_{2}$, $\chi_{6}=\phi_{3}$ and looking at the Poisson bracket matrix $\Delta_{i j}:=\left\{\chi_{i}, \chi_{j}\right\}$. On $\Sigma_{ \pm}$the components of the matrix are

$$
\Delta \approx\left(\begin{array}{cccccc}
0 & 0 & 0 & 2 i \hbar & \frac{ \pm p}{E}(i \hbar+2 \Delta(q p)) & \frac{ \pm 2 p}{E}(\Delta p)^{2} \\
0 & 0 & 0 & 0 & \frac{1}{2} i \hbar-\Delta(q p) & -(\Delta p)^{2} \\
0 & 0 & 0 & 0 & -(\Delta q)^{2} & -\frac{1}{2} i \hbar-\Delta(q p) \\
-2 i \hbar & 0 & 0 & 0 & 0 & 0 \\
\frac{\mp p}{E}(i \hbar+2 \Delta(q p)) & \Delta(q p)-\frac{1}{2} i \hbar & (\Delta q)^{2} & 0 & 0 & 0 \\
\frac{\mp 2 p}{E}(\Delta p)^{2} & (\Delta p)^{2} & \frac{1}{2} i \hbar+\Delta(q p) & 0 & 0 & 0
\end{array}\right) .
$$

Calculating the determinant one obtains the same result for both choices of the sign

$$
\operatorname{det}[\boldsymbol{\Delta}] \approx-4 \hbar^{2}\left[\frac{\hbar^{4}}{16}+(\Delta(q p))^{4}+2\left((\Delta p)^{2}(\Delta q)^{2}-\frac{\hbar^{2}}{4}\right)\left((\Delta p)^{2}(\Delta q)^{2}-(\Delta(q p))^{2}\right)\right]
$$

The determinant is non-zero in the region where reality, positivity and uncertainty conditions are imposed on the state - that is, if one demands

$$
\begin{aligned}
& q, p,(\Delta q)^{2}, \Delta(q p),(\Delta p)^{2} \in \mathbb{R} \\
& (\Delta p)^{2},(\Delta q)^{2} \geq 0 \\
& (\Delta p)^{2}(\Delta q)^{2}-(\Delta(q p))^{2} \geq \frac{\hbar^{2}}{4} .
\end{aligned}
$$

With these conditions in place, the sum of the terms inside the square bracket in the expression for the determinant is strictly positive, which means that the determinant itself is strictly negative.

There is one important check that one needs to perform. The introduction of $\phi_{i}=0$, $i=1,2,3$ makes the surfaces $\Sigma_{ \pm}$a mixture of first and second class and one is required to adjust the Poisson structure of the functions parameterizing the surfaces through the use of the Dirac bracket. Before we can identify $q, p,(\Delta q)^{2}, \Delta(q p),(\Delta p)^{2}$ as the expectation values and moments of a physical canonical pair of operators, we need to verify that their Dirac brackets on $\Sigma_{ \pm}$are identical to the Poisson brackets one would obtain for the 
quantum variables associated with a single canonical pair. The bracket may be computed as follows

$$
\{f, g\}_{\text {Dirac }}:=\{f, g\}-\left\{f, \chi_{i}\right\}\left(\Delta^{-1}\right)^{i j}\left\{\chi_{j}, g\right\} .
$$

Using the fact that $\Delta_{i j}$ (and hence also $\left(\Delta^{-1}\right)^{i j}$ ) is off-block-diagonal and that the physical variables have vanishing Poisson brackets with the gauge fixing conditions, one can easily verify that the second term in the above definition vanishes for the brackets between $q, p$, $(\Delta q)^{2}, \Delta(q p),(\Delta p)^{2}$ (as well as $t$ ), and therefore their Poisson structure is unchanged as required by our interpretation. These variables are the remaining physical quantities up to second order on the reduced quantum phase space.

To summarize, we impose the gauge-fixing conditions $\phi_{i}=0, i=1,2,3$, interpret $q$, $p,(\Delta q)^{2}, \Delta(q p),(\Delta p)^{2}$ as the physical expectation values and moments and as a result demand reality, positivity and quantum uncertainty. With all of these conditions taken together, $\phi_{i}=0, i=1,2,3$ restrict the gauge freedom up to the orbits generated by $C_{1 \pm}$ (recall that $C_{5 \pm}$ generates no flow on $\Sigma_{ \pm}$). This means that the time evolution is given by

$$
C_{\mathrm{Ham}}=\mu_{1} C_{1 \pm}
$$

Finally, we fix the remaining Lagrange multiplier $\mu_{1}$ by demanding $t$ - the last remaining gauge variable - to be the time parameter. That is, we demand that $\left\{t, C_{\mathrm{Ham}}\right\} \approx 1$, which leaves us with

$$
C_{\text {Ham }}=C_{1 \pm}=p_{t} \pm E .
$$

Taking the non-relativistic limit of $E$ we recover the results for a deparameterized free Newtonian particle. Specifically, if we formally take $p^{2} / m^{2}$ to be of order $\delta$, it follows that in a semiclassical state $(\Delta p)^{2} / m^{2}$ is of order $\hbar \delta$. We expand the expression for $E$ to the leading order in $\delta$ :

$$
\begin{aligned}
E & =\frac{m}{\sqrt{2}}\left(1+\frac{p^{2}+(\Delta p)^{2}}{m^{2}}\right)^{\frac{1}{2}}\left(1+\sqrt{1-\frac{4 p^{2}(\Delta p)^{2}}{\left(p^{2}+m^{2}+(\Delta p)^{2}\right)^{2}}}\right)^{\frac{1}{2}} \\
& =\frac{m}{\sqrt{2}}\left(1+\frac{p^{2}+(\Delta p)^{2}}{2 m^{2}}+O\left(\delta^{2}\right)\right)\left(2+O\left(\hbar \delta^{2}\right)\right)^{\frac{1}{2}} \\
& =m+\frac{p^{2}+(\Delta p)^{2}}{2 m}+O\left(\delta^{2}\right) .
\end{aligned}
$$

\subsection{Comparison with the Klein-Gordon solution}

The standard positive frequency solutions to the Klein-Gordon equation (see for example [18]) form a Hilbert space of momentum-space wave-functions square integrable with respect to the Lorentz-invariant measure:

$$
\mathcal{H}=L^{2}\left(\mathbb{R}, \frac{\mathrm{d} k}{2 \epsilon_{k}}\right), \quad \text { where } \quad \epsilon_{k}=\sqrt{k^{2}+m^{2}}
$$


The system can be described through a canonical pair of observables, represented on $\mathcal{H}$ as

$$
\mathbf{p}=k \quad \text { and } \quad \mathbf{q}=i \hbar\left(\frac{\partial}{\partial k}+\epsilon_{k}\left(\frac{\partial}{\partial k} \frac{1}{2 \epsilon_{k}}\right)\right)
$$

The time evolution is generated by the Hamiltonian $\mathbf{H}=\left(\mathbf{p}^{2}+m^{2} \mathbb{1}\right)^{\frac{1}{2}}$. One can evaluate the evolution equations for the expectation values of the observables using Ehrenfest's theorem

$$
\frac{\mathrm{d}}{\mathrm{d} t}\langle\mathbf{O}\rangle=\frac{1}{i \hbar}\langle[\mathbf{O}, \mathbf{H}]\rangle+\frac{\partial\langle\mathbf{O}\rangle}{\partial t} .
$$

In our formalism, the right-hand side is equivalent to the quantum Poisson bracket between the expectation values, thus

$$
\frac{\mathrm{d}}{\mathrm{d} t}\langle\mathbf{O}\rangle=\{\langle\mathbf{O}\rangle,\langle\mathbf{H}\rangle\}+\frac{\partial\langle\mathbf{O}\rangle}{\partial t}=\left\{\langle\mathbf{O}\rangle,\left\langle\left(\mathbf{p}^{2}+m^{2} \mathbb{1}\right)^{\frac{1}{2}}\right\rangle\right\}+\frac{\partial\langle\mathbf{O}\rangle}{\partial t} .
$$

We recall that our procedure at order $\hbar$ together with the gauge fixing conditions for the positive frequency solutions resulted in

$$
\frac{\mathrm{d}}{\mathrm{d} t}\langle\mathbf{O}\rangle=\left\{\langle\mathbf{O}\rangle, p_{t}+E\right\}=\{\langle\mathbf{O}\rangle, E\}+\frac{\partial\langle\mathbf{O}\rangle}{\partial t} .
$$

In order to see whether the methods agree, we only need to compare $\left\langle\left(\mathbf{p}^{2}+m^{2} \mathbb{1}\right)^{\frac{1}{2}}\right\rangle$ and $E$ to order $\hbar$. To verify this explicitly we expand the operator in terms of its moments, assuming the expectation value to be taken in a semiclassical state:

$$
\begin{aligned}
\left\langle\left(\mathbf{p}^{2}+m^{2} \mathbb{1}\right)^{\frac{1}{2}}\right\rangle & \left.=\left\langle\left(p^{2}+m^{2}\right)^{\frac{1}{2}}+\frac{p}{\left(p^{2}+m^{2}\right)^{\frac{1}{2}}}(\mathbf{p}-p)+\frac{m^{2}}{2\left(p^{2}+m^{2}\right)^{\frac{3}{2}}}(\mathbf{p}-p)^{2}\right\rangle+\text { (higher moments }\right) \\
& =\sqrt{p^{2}+m^{2}}\left(1+\frac{m^{2}(\Delta p)^{2}}{2\left(p^{2}+m^{2}\right)^{2}}\right)+O\left(\hbar^{\frac{3}{2}}\right) .
\end{aligned}
$$

For comparison, we expand $E$ in powers of $(\Delta p)^{2}$, which we assume to be of order $\hbar$.

$$
\begin{aligned}
E & =\frac{1}{\sqrt{2}} \sqrt{p^{2}+m^{2}}\left(1+\frac{(\Delta p)^{2}}{p^{2}+m^{2}}+1+\frac{\left(m^{2}-p^{2}\right)(\Delta p)^{2}}{\left(p^{2}+m^{2}\right)^{2}}+O\left((\Delta p)^{4}\right)\right)^{\frac{1}{2}} \\
& =\sqrt{p^{2}+m^{2}} \sqrt{1+\frac{m^{2}(\Delta p)^{2}}{\left(p^{2}+m^{2}\right)^{2}}+O\left((\Delta p)^{4}\right)} \\
& =\sqrt{p^{2}+m^{2}}\left(1+\frac{m^{2}(\Delta p)^{2}}{2\left(p^{2}+m^{2}\right)^{2}}\right)+O\left((\Delta p)^{4}\right) .
\end{aligned}
$$

Thus, up to the terms of order $\hbar$ the two results agree.

Unlike the exact Klein-Gordon solution, our approach avoids explicit reference to a representation. The action of the Lorentz group on our variables can be understood through 
its action on the algebra of observables. In particular, we assume that the pairs $\left(\mathbf{p}_{t}, \mathbf{p}\right)$ and $(\mathbf{t}, \mathbf{q})$ transform as components of a contravariant and a covariant vector respectively. Looking at the truncated system of constraints (5) under a Lorentz transformation one finds that $C$ remains invariant, while the pairs $\left(C_{p_{t}}, C_{p}\right)$ and $\left(C_{t}, C_{q}\right)$ themselves transform as components of a contravariant and a covariant vector respectively, so that the whole truncated system of constraints is preserved.

\subsection{Free massless particle}

For a massless particle, the constraint operator takes the form

$$
\mathbf{C}=\mathbf{p}_{t}^{2}-\mathbf{p}^{2}
$$

To second order in moments, the constraint functions produced remain as in equation (5), except for $C$, which now reads

$$
C=\langle\mathbf{C}\rangle=p_{t}^{2}-p^{2}+\left(\Delta p_{t}\right)^{2}-(\Delta p)^{2}=0
$$

The disappearance of a constant term from $C$ does not affect the Poisson algebra of the constraints, so that the table of Section 3.1 still applies. The solution to the constraints, however takes on a simpler form: following the same steps as previously and eliminating $\left(\Delta p_{t}\right)^{2}$ in a way compatible with the semiclassical approximation we obtain

$$
\left(\Delta p_{t}\right)^{2}=(\Delta p)^{2}
$$

Together with $C=0$ this implies

$$
p_{t}^{2}=p^{2}
$$

As we see, the classical constraint is satisfied exactly by the expectation values. We solve this via

$$
p_{t}= \pm|p|
$$

There are two related reasons for taking the absolute value of $p$ in the above solution. Firstly, to emphasize the sign of the energy. Secondly, to match the limit as $m$ is set to zero of the solution obtained in Section 3.1. The full solutions read

$$
\begin{aligned}
p_{t} & = \pm|p| \\
\Delta\left(t p_{t}\right) & = \pm \frac{p}{|p|} \Delta(t p)-\frac{i \hbar}{2} \\
\left(\Delta p_{t}\right)^{2} & =(\Delta p)^{2} \\
\Delta\left(p_{t} q\right) & = \pm \frac{p}{|p|}\left(\Delta(q p)+\frac{i \hbar}{2}\right) \\
\Delta\left(p_{t} p\right) & = \pm \frac{p}{|p|}(\Delta p)^{2} .
\end{aligned}
$$


The steps of Section 3.2 can be repeated exactly for the $m=0$ case with $|p|$ playing the role of $E$. With the gauges fixed in an identical way, this results in evolution on $q, p$, $(\Delta q)^{2}, \Delta(q p),(\Delta p)^{2}$ generated by the constraint

$$
C_{\text {Наm }}=p_{t} \pm|p| .
$$

The implications will be discussed further in the conclusions.

\section{Relativistic particle in a potential}

In this section we consider the consequences of adding a potential term to the quantum constraint. We consider a quadratic time-independent potential in Section 4.1 followed by a homogeneous time-dependent potential in Section 4.2. The systems considered in this section have the same kinematical degrees of freedom as the free relativistic particle; however, the additional terms in the constraint element break Lorentz invariance. On the other hand, certain structural properties of the constraint element remain very similar to the free particle case, which makes extension of the calculations performed in Section 3 fairly simple. As we will see, the constraints are still straightforward to solve, but their Poisson algebra is only approximately closed and in the case of the time-dependent potential, the gauge analysis requires more subtlety. These examples show that the effective procedure used here is feasibly applicable to a wider range of models than the existing explicit constructions of a physical inner product.

\subsection{Quadratic potential}

A relativistic particle in a quadratic potential is subject to the constraint

$$
\mathbf{C}=\mathbf{p}_{t}^{2}-\mathbf{p}^{2}-\mathbf{q}^{2}-m^{2} \mathbb{1} .
$$

(A coupling constant in the potential could be absorbed by rescaling.) This gives rise to the following set of constraint functions truncated at second order

$$
\begin{aligned}
C & =p_{t}^{2}-p^{2}-q^{2}-m^{2}+\left(\Delta p_{t}\right)^{2}-(\Delta p)^{2}-(\Delta q)^{2}=0 \\
C_{t} & =2 p_{t} \Delta\left(t p_{t}\right)+i \hbar p_{t}-2 p \Delta(t p)-2 q \Delta(t q)=0 \\
C_{p_{t}} & =2 p_{t}\left(\Delta p_{t}\right)^{2}-2 p \Delta\left(p_{t} p\right)-2 q \Delta\left(p_{t} q\right)=0 \\
C_{q} & =2 p_{t} \Delta\left(p_{t} q\right)-2 p \Delta(q p)-i \hbar p-2 q(\Delta q)^{2}=0 \\
C_{p} & =2 p_{t} \Delta\left(p_{t} p\right)-2 p(\Delta p)^{2}-2 q \Delta(q p)+i \hbar q=0 .
\end{aligned}
$$

The above system of constraints is first class only to order $\hbar$ as can be seen from their Poisson algebra in Table 2,

The system of constraints may be solved following the same steps that have been employed to solve the constraints for the free particle. We use $C, C_{q}$, and $C_{p}$ to eliminate 
Table 2: Poisson algebra of constraints for the particle in a quadratic potential. First terms in the bracket are labeled by rows, second terms are labeled by columns.

\begin{tabular}{|c|c|c|c|c|c|}
\hline & $C$ & $C_{t}$ & $C_{p_{t}}$ & $C_{q}$ & $C_{p}$ \\
\hline$C$ & 0 & $-2 C_{p_{t}}$ & 0 & $2 C_{p}$ & $-2 C_{q}$ \\
\hline$C_{t}$ & $2 C_{p_{t}}$ & 0 & $\begin{array}{c}4 p_{t} C_{p_{t}}-2 p C_{p}-2 q C_{q} \\
+4 \Delta\left(p_{t} p\right) \Delta(t q) \\
-4 \Delta\left(p_{t} q\right) \Delta(t p) \\
\end{array}$ & $\begin{array}{c}2 p_{t} C_{q}+2 p C_{t} \\
+4 \Delta(t q)\left(\Delta(q p)+\frac{i \hbar}{2}\right) \\
+4(\Delta q)^{2} \Delta(t p) \\
\end{array}$ & $\begin{array}{c}2 p_{t} C_{p}+2 q C_{t} \\
+4 \Delta(t q)(\Delta p)^{2} \\
-4 \Delta(t p)\left(\Delta(q p)-\frac{i \hbar}{2}\right)\end{array}$ \\
\hline$C_{p_{t}}$ & 0 & $\begin{array}{c}-4 p_{t} C_{p_{t}}+2 p C_{p}+2 q C_{q} \\
-4 \Delta\left(p_{t} p\right) \Delta(t q) \\
+4 \Delta\left(p_{t} q\right) \Delta(t p) \\
\end{array}$ & 0 & $\begin{array}{c}2 p C_{p_{t}} \\
-4(\Delta q)^{2} \Delta\left(p_{t} p\right) \\
-\Delta\left(p_{t} q\right)\left(\Delta(q p)-\frac{i \hbar}{2}\right) \\
\end{array}$ & $\begin{array}{c}-2 q C_{p_{t}} \\
+4(\Delta p)^{2} \Delta\left(p_{t} q\right) \\
+4 \Delta\left(p_{t} p\right)\left(\Delta(q p)-\frac{i \hbar}{2}\right)\end{array}$ \\
\hline$C_{q}$ & $-2 C_{p}$ & $\begin{array}{c}-2 p t C_{q}-2 p C_{t} \\
-4 \Delta(t q)\left(\Delta(q p)+\frac{i \hbar}{2}\right) \\
-4 \Delta(t p)(\Delta q)^{2} \\
\end{array}$ & $\begin{array}{c}-2 p C_{p_{t}} \\
+4(\Delta q)^{2} \Delta\left(p_{t} p\right) \\
+4 \Delta\left(p_{t} q\right)\left(\Delta(q p)-\frac{i \hbar}{2}\right)\end{array}$ & 0 & $\begin{array}{c}2 p_{t} C_{p_{t}}-4 p C_{p}-4 q C_{q} \\
+4\left((\Delta q)^{2}(\Delta p)^{2}-\frac{\hbar^{2}}{4}\right) \\
-4(\Delta(q p))^{2}\end{array}$ \\
\hline$C_{p}$ & $2 C_{q}$ & $\begin{array}{c}-2 p_{t} C_{p}+2 q C_{t} \\
-4 \Delta(t q)(\Delta p)^{2} \\
+4 \Delta(t p)\left(\Delta(q p)-\frac{i \hbar}{2}\right)\end{array}$ & $\begin{array}{c}2 q C_{p_{t}} \\
-4(\Delta p)^{2} \Delta\left(p_{t} q\right) \\
+4 \Delta\left(p_{t} p\right)\left(\Delta(q p)-\frac{i \hbar}{2}\right)\end{array}$ & $\begin{array}{c}4 p C_{p}+4 q C_{q}-2 p_{t} C_{p_{t}} \\
-4\left((\Delta q)^{2}(\Delta p)^{2}-\frac{\hbar^{2}}{4}\right) \\
-4(\Delta(q p))^{2}\end{array}$ & 0 \\
\hline
\end{tabular}

$p_{t}^{2}, \Delta\left(p_{t} q\right)$ and $\Delta\left(p_{t} p\right)$ respectively. Substituted into $C_{p_{t}}$ this yields a quadratic equation in $\left(\Delta p_{t}\right)^{2}$ :

$0=\left(\left(\Delta p_{t}\right)^{2}\right)^{2}-\left(p^{2}+q^{2}+m^{2}+(\Delta p)^{2}+(\Delta q)^{2}\right)\left(\Delta p_{t}\right)^{2}+\left(p^{2}(\Delta p)^{2}+2 q p \Delta(q p)+q^{2}(\Delta q)^{2}\right)$.

The solution compatible with the semiclassical approximation has the form

$$
\begin{aligned}
p_{t} & = \pm E \\
\Delta\left(t p_{t}\right) & =\frac{ \pm 1}{E}(p \Delta(t p)+q \Delta(t q))-\frac{i \hbar}{2} \\
\left(\Delta p_{t}\right)^{2} & =p^{2}+q^{2}+m^{2}+(\Delta p)^{2}+(\Delta q)^{2}-E^{2} \\
\Delta\left(p_{t} q\right) & =\frac{ \pm 1}{E}\left(p \Delta(q p)+\frac{i \hbar}{2} p+q(\Delta q)^{2}\right) \\
\Delta\left(p_{t} p\right) & =\frac{ \pm 1}{E}\left(p(\Delta p)^{2}+q \Delta(q p)-\frac{i \hbar}{2} q\right)
\end{aligned}
$$

where

$$
\begin{aligned}
E= & \frac{1}{\sqrt{2}}\left[p^{2}+q^{2}+m^{2}+(\Delta p)^{2}+(\Delta q)^{2}\right. \\
& \left.+\left(\left(p^{2}+q^{2}+m^{2}+(\Delta p)^{2}+(\Delta q)^{2}\right)^{2}-4\left(p^{2}(\Delta p)^{2}+2 q p \Delta(q p)+q^{2}(\Delta q)^{2}\right)\right)^{\frac{1}{2}}\right]^{\frac{1}{2}}
\end{aligned}
$$

We note that as $C=p_{t}^{2}-E^{2}$ and $p_{t}$ are both exactly first class, $E$ must also be first class, since it does not vanish on the constraint surface. 
If one applies the semiclassical approximation once again to drop the terms of orders higher than $\hbar$, the constraint system may be treated as first class. The linearized versions of the constraints take the form

$$
\begin{aligned}
& C_{1 \pm}=p_{t} \pm E \\
& C_{2 \pm}=\left(\Delta p_{t}\right)^{2}-p^{2}-q^{2}-m^{2}-(\Delta p)^{2}-(\Delta q)^{2}+E^{2} \\
& C_{3 \pm}=\Delta\left(p_{t} p\right) \pm \frac{1}{E}\left(p(\Delta p)^{2}+q \Delta(q p)-q \frac{i \hbar}{2}\right) \\
& C_{4 \pm}=\Delta\left(p_{t} q\right) \pm \frac{1}{E}\left(p \Delta(q p)+p \frac{i \hbar}{2}+q(\Delta q)^{2}\right) \\
& C_{5 \pm}=\Delta\left(t p_{t}\right) \pm \frac{1}{E}(p \Delta(t p)+q \Delta(t q))+\frac{i \hbar}{2} .
\end{aligned}
$$

One can follow the process outlined in the Section 3.2 and impose the set of gauge-fixing conditions (10). Once again $C_{1 \pm}$ is first-class on the gauge-fixed surfaces $\Sigma_{ \pm}$and writing $C_{5 \pm}=\Delta\left(t p_{t}\right) \pm \frac{p}{E} \phi_{3} \pm \frac{q}{E} \phi_{2}$ it is not difficult to see that, once again, $C_{5 \pm}$ remains first-class but has a vanishing flow on $\Sigma_{ \pm}$. We recall that the gauge conditions have a vanishing flow on the remaining free variables; therefore only the first term in the expressions for each of the constraints $C_{i \pm}$ above has a non-vanishing Poisson bracket with the conditions $\phi_{i}$. As a result, the Poisson bracket matrix $\Delta$ remains as in Section 3.2 up to entries of order $\hbar$. Imposing reality, positivity and quantum uncertainty and demanding $\left\{t, C_{\text {Ham }}\right\} \approx 1$ we once again obtain

$$
C_{\mathrm{Ham}}=p_{t} \pm E
$$

Directly expanding $E$ in powers of $(\Delta q)^{2}, \Delta(q p)$ and $(\Delta p)^{2}$ we get the expression to order $\hbar$

$$
\begin{aligned}
E=\sqrt{p^{2}+q^{2}+m^{2}}\left[1+\frac{\left(q^{2}+m^{2}\right)(\Delta p)^{2}-2 q p \Delta(q p)+\left(p^{2}+m^{2}\right)(\Delta q)^{2}}{2\left(p^{2}+q^{2}+m^{2}\right)^{2}}\right] \\
+O\left((\Delta p)^{4}\right)+O\left((\Delta q)^{4}\right)+O\left((\Delta(q p))^{2}\right) .
\end{aligned}
$$

The specific constraint considered in this section can be implemented quite completely at the level of physical states, although specifics of the dynamics are more difficult to extract than with effective methods. Below we briefly describe the solution and compare it to our effective treatment. The algebra elements may be represented kinematically as differential operators on the space of square-integrable wave-functions in two variables $x_{0}$ and $x_{1}$ in the usual way

$$
\mathbf{t}=x_{0}, \quad \mathbf{p}_{t}=\frac{\hbar}{i} \frac{\partial}{\partial x_{0}}, \quad \mathbf{q}=x_{1}, \quad \mathbf{p}=\frac{\hbar}{i} \frac{\partial}{\partial x_{1}}
$$

The constraint operator splits into a sum of commuting, and therefore simultaneously diagonalizable, components: 
- $\mathbf{p}_{t}^{2}=-\hbar^{2} \frac{\partial^{2}}{\partial x_{0}^{2}}$ has infinite-norm eigenstates of the form $\exp \left(\frac{i k}{\hbar} x_{0}\right) \phi\left(x_{1}\right)$, with eigenvalues $k^{2}$.

- $\mathbf{p}^{2}+\mathbf{q}^{2}=-\hbar^{2} \frac{\partial^{2}}{\partial x_{1}^{2}}+x_{1}^{2}=2 \mathbf{H}_{\text {harm }}$, where $\mathbf{H}_{\text {harm }}$ is precisely the standard Hamiltonian of the harmonic oscillator on $x_{1}$ (with mass and frequency set to unity). This operator has normalizable eigenstates of the form $\psi\left(x_{0}\right) \varphi_{n}\left(x_{1}\right)$, where $\varphi_{n}\left(x_{1}\right)$ is the usual normalized $n$-th eigenstate of the harmonic oscillator, the corresponding eigenvalues are $2\left(n+\frac{1}{2}\right) \hbar$.

- Every wavefunction is an eigenstate of $1 m^{2}$ with the eigenvalue $m^{2}$.

The eigenfunctions of the constraint operator are $\Psi_{k, n}\left(x_{0}, x_{1}\right)=\exp \left(\frac{i k}{\hbar} x_{0}\right) \varphi_{n}\left(x_{1}\right)$, the corresponding eigenvalues are $\left(k^{2}-2\left(n+\frac{1}{2}\right) \hbar-m^{2}\right)$. The space of solutions to the quantum constraint equation is therefore spanned by the wavefunctions $\Psi_{k, n}$ for which $k=$ $\pm \sqrt{2\left(n+\frac{1}{2}\right) \hbar+m^{2}}$. These states are not normalizable with respect to the square integration in both $x_{0}$ and $x_{1}$, however they have unit norm when the integration is taken with respect to $x_{1}$ alone.

As is usually done for such systems we decompose the solution space into two segments one belongs to the positive part, the other one to the negative part of the spectrum of $\mathbf{p}_{t}$ (e.g. the separation of positive and negative frequencies of the solutions to the KleinGordon equation). The general element of the solution space is a linear combination of either positive or negative frequency null eigenfunctions of $\mathbf{C}$, denoted by $\Psi^{+}$and $\Psi^{-}$ respectively

$$
\Psi_{\mathrm{phys}}^{ \pm}=\sum_{n=0}^{\infty} \alpha_{n} \exp \left(\frac{\mp i x_{0} \sqrt{2\left(n+\frac{1}{2}\right) \hbar+m^{2}}}{\hbar}\right) \varphi_{n}\left(x_{1}\right) .
$$

The separation into two components allows us to define a positive-definite physical inner product on each one of them individually

$$
\langle\Psi \mid \Phi\rangle_{\text {phys }}:=\int_{-\infty}^{\infty} \overline{\Psi\left(x_{0}, x_{1}\right)} \Phi\left(x_{0}, x_{1}\right) \mathrm{d} x_{1}
$$

where the bar denotes a complex-conjugate and both states $\Psi, \Phi$ belong to the same component. On the space of solutions, the above inner product is independent of the value taken by $x_{0}$. Furthermore this inner product is consistent with interpreting $\mathbf{t}$ as time, since we can formally write $\langle\Psi|\mathbf{t}| \Psi\rangle_{\text {phys }}=x_{0}$. (This equation requires some care in its interpretation since $\mathbf{t}$ is not a physical operator. But just viewing the integration on the right hand side of (19) easily allows us to introduce an operator $\mathbf{t}$ by multiplication. The dependence on $x_{0}$ of the result is then in agreement with the fact that $\mathbf{t}$ is not a physical observable.) It is also consistent with the gauge choices of equation (10), which is straightforward to verify using the fact that for any operator $\mathbf{A}$, polynomial in $\mathbf{t}, \mathbf{p}_{t}, \mathbf{q}, \mathbf{p}$

$$
\langle\Psi|\mathbf{t} \mathbf{A}| \Psi\rangle_{\text {phys }}=x_{0}\langle\Psi|\mathbf{A}| \Psi\rangle_{\text {phys }} .
$$


The physical states may be interpreted as solutions of one of the two Scrödinger equations

$$
-\frac{\hbar}{i} \frac{\mathrm{d}}{\mathrm{d} x_{0}} \Psi^{ \pm}= \pm\left(\mathbf{p}^{2}+\mathbf{q}^{2}+m^{2} \mathbb{1}\right)^{\frac{1}{2}} \Psi^{ \pm}
$$

That is, an ordinary quantum mechanical system with time evolution in the variable $x_{0}$ generated by the self-adjoint, positive square-root Hamiltonian $\mathbf{H}=\left(\mathbf{p}^{2}+\mathbf{q}^{2}+m^{2} \mathbb{1}\right)^{\frac{1}{2}}$, which is defined through its action on the basis of eigenstates: $\mathbf{H} \varphi_{n}\left(x_{1}\right)=\sqrt{2\left(n+\frac{1}{2}\right) \hbar+m^{2}} \varphi_{n}\left(x_{1}\right)$.

To compare the physical states and the effective solutions, we proceed as we have done before, in Section 3.3. We expand the expectation value of the square-root hamiltonian in a semiclassical state

$$
\begin{aligned}
\langle\mathbf{H}\rangle= & \left\langle\left\langle\mathbf{p}^{2}+\mathbf{q}^{2}+m^{2} \mathbb{1}\right\rangle^{\frac{1}{2}}+\frac{\left(\mathbf{p}^{2}+\mathbf{q}^{2}+m^{2} \mathbb{1}\right)-\left\langle\mathbf{p}^{2}+\mathbf{q}^{2}+m^{2} \mathbb{1}\right\rangle}{2\left\langle\mathbf{p}^{2}+\mathbf{q}^{2}+m^{2} \mathbb{1}\right\rangle^{\frac{1}{2}}}\right. \\
& \left.\left.-\frac{\left(\left(\mathbf{p}^{2}+\mathbf{q}^{2}+m^{2} \mathbb{1}\right)-\left\langle\mathbf{p}^{2}+\mathbf{q}^{2}+m^{2} \mathbb{1}\right\rangle\right)^{2}}{8\left\langle\mathbf{p}^{2}+\mathbf{q}^{2}+m^{2} \mathbb{1}\right\rangle^{\frac{3}{2}}}\right\rangle+ \text { (higher moments }\right) .
\end{aligned}
$$

Proceeding with the above expansion and keeping only the terms up to order $\hbar$ one obtains the expression that is identical to the one for $E$ in equation (18). Thus, to leading order in the semiclassical regime, the effective solution to the constraint is consistent with physical state evolution, and the gauge choice of equation (10) is consistent with the physical inner product defined above together with the interpretation of $\langle\mathbf{t}\rangle$ as measuring the physical time. For a direct comparison between fully quantum and effective time evolutions for a specific semiclassical state of this system see App. B.

If we replace $\left(\mathbf{p}^{2}+\mathbf{q}^{2}+m^{2} \mathbb{1}\right)$ in the constraint by any positive operator $f(\mathbf{q}, \mathbf{p})$ analytic in $\mathbf{q}$ and $\mathbf{p}$, physical states can in principle be found in a similar way. One could find the spectrum of $f(\mathbf{q}, \mathbf{p})$ and construct the solutions out of its simultaneous eigenfunctions with $\mathbf{p}_{t}^{2}$. Finding the spectrum of a given operator is in general a complicated task. Further, we were helped in this example by the fact that the spectrum of $\left(\mathbf{p}^{2}+\mathbf{q}^{2}+m^{2} \mathbb{1}\right)$ is discrete and the eigenfunctions are normalizable with respect to square integration over $x_{1}$ alone. Defining the physical inner product is more difficult if parts of the spectrum of $f(\mathbf{q}, \mathbf{p})$ are continuous. Finally, determining suitable coherent states for semiclassical purposes, as done for this model in App. B can be a difficult task. The leading order effective solution, on the other hand, can be obtained in much the same way as was done for the above example, without explicit in-depth knowledge of the spectrum of $\mathbf{q}$ or the exact form of its eigenstates.

\subsection{Time-dependent potential}

As mentioned in the introduction, time-dependent potentials are of interest in quantum cosmology. Another example where time-dependent terms arise is a relativistic particle moving on a non-static curved background space-time. In such cases, our methods can be 
used as well, but additional subtleties do arise. Adding a "potential" $V(t)=\lambda t$ to the classical constraint gives the second order quantum constraints

$$
\begin{aligned}
C & =p_{t}^{2}-p^{2}-m^{2}+\left(\Delta p_{t}\right)^{2}-(\Delta p)^{2}+\lambda t=0 \\
C_{t} & =2 p_{t} \Delta\left(t p_{t}\right)+i \hbar p_{t}-2 p \Delta(t p)+\lambda(\Delta t)^{2}=0 \\
C_{p_{t}} & =2 p_{t}\left(\Delta p_{t}\right)^{2}-2 p \Delta\left(p_{t} p\right)+\lambda \Delta\left(p_{t} t\right)-\frac{1}{2} i \lambda \hbar=0 \\
C_{q} & =2 p_{t} \Delta\left(p_{t} q\right)-2 p \Delta(q p)-i \hbar p+\lambda \Delta(q t)=0 \\
C_{p} & =2 p_{t} \Delta\left(p_{t} p\right)-2 p(\Delta p)^{2}+\lambda \Delta(t p)=0 .
\end{aligned}
$$

These constraints, once again, form a closed Poisson algebra only up to order $\hbar$. We proceed to solve the above set of polynomial equations explicitly $-p_{t} C_{p_{t}}=0$ implies

$$
p_{t}^{2}\left(\Delta p_{t}\right)^{2}-p_{t} p \Delta\left(p_{t} p\right)+\frac{1}{2} \lambda p_{t} \Delta\left(t p_{t}\right)-\frac{i \hbar}{4} \lambda p_{t}=0 .
$$

Using $p C_{p}=0$ and $\lambda C_{t}=0$ to eliminate $p_{t} p \Delta\left(p_{t} p\right)$ and $\lambda p_{t} \Delta\left(t p_{t}\right)$ respectively we obtain

$$
p_{t}^{2}\left(\Delta p_{t}\right)^{2}-p^{2}(\Delta p)^{2}+\lambda p \Delta(t p)-\frac{1}{4} \lambda^{2}(\Delta t)^{2}-\frac{i \hbar}{2} \lambda p_{t}=0 .
$$

Finally, we eliminate $\left(\Delta p_{t}\right)^{2}$ using $C=0$ to obtain a quartic equation in $p_{t}$

$$
0=p_{t}^{4}-\left(p^{2}+m^{2}-\lambda t+(\Delta p)^{2}\right) p_{t}^{2}+\frac{i \hbar}{2} \lambda p_{t}+\left(p^{2}(\Delta p)^{2}+\frac{1}{4} \lambda^{2}(\Delta t)^{2}-\lambda p \Delta(t p)\right) .
$$

The exact solutions to the above quartic equation are of course readily available, though they involve long algebraic expressions and are not particularly illuminating. Furthermore, due to the linear term in $p_{t}$ in the equation, the gauge choices we have employed previously together with the reality conditions would lead us to conclude that $p_{t}$ is complex. A more subtle gauge analysis is required to solve the constraint without further approximations. For instance, to respect reality conditions one would use a gauge relating moments to the expectation values, for example by making $\Delta(t p)$ dependent on $p_{t}$.

However, assuming the potential changes very slowly allows one to move forward with the standard gauge choice. Treating $\lambda$ as a second small parameter in addition to $\hbar^{1 / 2}$, such that $\lambda \hbar$ is of higher than second order and discarding the terms of order higher than $\hbar$ we are left with

$$
p_{t}^{4}-\left(p^{2}+m^{2}-\lambda t+(\Delta p)^{2}\right) p_{t}^{2}+p^{2}(\Delta p)^{2}=0 .
$$

This equation could also be obtained by directly dropping products of $\lambda$ and second order moments or $\hbar$ in the expressions for the constraint functions (20) and solving them. It is a quadratic equation in $p_{t}^{2}$, with solutions that are much easier to interpret. Compatibility with the semiclassical approximation once again selects for us a set of solutions that have a very similar form to those in (7), (12), and (15), with

$$
E=\frac{1}{\sqrt{2}}\left[p^{2}+m^{2}-\lambda t+(\Delta p)^{2}+\left(\left(p^{2}+m^{2}-\lambda t+(\Delta p)^{2}\right)^{2}-4 p^{2}(\Delta p)^{2}\right)^{\frac{1}{2}}\right]^{\frac{1}{2}}
$$


One can then repeat the gauge-fixing procedure we have previously employed and recover $C_{\text {Ham }}=p_{t} \pm E$, for a slowly varying potential in a semiclassical state. Thus, to the semiclassical order considered, the system behaves as a non-relativistic quantum particle in one dimension subject to a time-dependent hamiltonian $\mathbf{H}=\left(\mathbf{p}^{2}+m^{2} \mathbb{1}-\lambda t\right)^{\frac{1}{2}}$.

In fact, a more general "slowly varying" potential may be treated to order $\hbar$ in an analogous manner. We assume that the potential has the form $V(\mathbf{t})=V(0) \mathbb{1}+\lambda \tilde{V}(\mathbf{t})$, where $\lambda$ is small in the sense discussed earlier, and $\tilde{V}(\mathbf{t})$ is a polynomial in $\mathbf{t}$. This implies, in particular, that $\langle\lambda \tilde{V}(\mathbf{t})\rangle=\lambda \tilde{V}(t)+O\left(\hbar^{\frac{3}{2}}\right)$. We absorb the constant part of the potential into the definition of $m$ and the constraint functions to order $\hbar$ look exactly as they do for a free relativistic particle (5), except for $C$, which acquires an extra term

$$
C=p_{t}^{2}-p^{2}-m^{2}+\left(\Delta p_{t}\right)^{2}-(\Delta p)^{2}+\lambda \tilde{V}(t)=0 .
$$

The resulting system of constraint functions may be solved, gauge-fixed and interpreted directly following the methods employed throughout Sections 3 and 4 . This demonstrates the flexibility of the constructions, confirming the methods of [11, 12, 13], where also slowly varying potentials were assumed. In contrast to this earlier work, the general methods presented here are in principle applicable to arbitrary potentials, but the gauge fixing would have to be considered in each case in detail. This provides access to questions about the role of time when potentials forbid a global monotonic internal time $t$, resulting in a new perspective to be followed elsewhere.

\section{Conclusion}

One of the main hurdles for quantum gravity is the physical Hilbert space issue. At least for semiclassical questions, technical and conceptual difficulties can be circumvented by using expectation values and moments directly rather than states. Other advantages of this method are that the specification of semiclassical regimes is easier via moments (while semiclassical wave functions are often difficult to formulate, even simple-looking Gaussian ones not always being semiclassical at all in some models of quantum cosmology as pointed out in [19]) and that density states are automatically included.

We have extended the methods for effective constraints of [2] to relativistic systems, clarifying several physically relevant issues of effective equations:

- Square root effective Hamiltonians, which so far were strictly justified only for timeindependent potentials, are valid even in the time dependent case provided the potential varies slowly in time. No extra conditions on the dynamics are implied by the positivity conditions.

- Quantum back-reaction follows reliably from square-root Hamiltonians. Our examples of relativistic systems have shown three different cases:

- Massless particles do not contain moments in their reduced Hamiltonian (13) and thus are not subject to quantum back-reaction. 
- Free massive particles do have quantum back-reaction from moments of all orders, which is initially unexpected since the quantum constraint has only a linear term of $(\Delta p)^{2}$ with no coupling to the expectation values. (Our formulas, done here only to second order, do not show this explicitly.)

- Particles subject to a $q$-dependent potential receive quantum back-reaction from the covariance of their wave function as seen in (16). This is also unexpected since the expectation value of the constraint does not contain mixed moments. As in the case of massive particles, the unexpected results are explained by the presence of higher order constraints.

- Higher order constraints, which are crucial for the effective procedure, also affect the amount of spreading of wave functions, or the time dependence of moments. For a free, massless particle wave packets do not spread, but they do in the other cases.

Effective equations obtained in the way developed here reliably describe the physical behavior of dynamical wave packets. With these considerations, effective methods as they have been used in quantum cosmology are established even in the case of $\phi$-dependent potentials, as studied specifically for instance in [13, 8]. A further application would be to reconsider the appearance of certain future singularities which have been shown not to be removed by the tree-level approximation (disregarding all moments) [20] but where quantum back-reaction is expected to be strong.

Especially in the presence of potentials, calculations performed here are much more feasible than the methods involving constructions of physical Hilbert spaces followed by computations of the expectation values in explicit physical states. They can be expected to be of far more general applicability, including full quantum gravity. For such an extension, several other issues remain open: describing field theories and handling situations of many classical degrees of freedom. But there is already a promise that effective techniques allow one to evade difficult obstacles from physical inner product issues which so far have impeded progress. Especially the semiclassical regime of canonical quantum gravity and potentially observable effects can be brought under much higher control.

\section{Acknowledgements}

We thank Don Marolf and Madhavan Varadarajan for discussions. This work was supported in part by NSF grant PHY0748336.

\section{A Second order Poisson algebra}

The expectation values obey the classical Poisson algebra for two canonical pairs, where the non trivial brackets are

$$
\left\{t, p_{t}\right\}=1 \quad \text { and } \quad\{q, p\}=1 .
$$


Table 3: Poisson algebra of second order moments. First terms in the bracket are labeled by rows, second terms are labeled by columns.

\begin{tabular}{|c||c|c|c|c|c|c|c|c|c|c|}
\hline & $(\Delta t)^{2}$ & $\Delta\left(t p_{t}\right)$ & $\left(\Delta p_{t}\right)^{2}$ & $(\Delta q)^{2}$ & $\Delta(q p)$ & $(\Delta p)^{2}$ & $\Delta(t q)$ & $\Delta\left(p_{t} p\right)$ & $\Delta(t p)$ & $\Delta\left(p_{t} q\right)$ \\
\hline \hline$(\Delta t)^{2}$ & 0 & $2(\Delta t)^{2}$ & $4 \Delta\left(t p_{t}\right)$ & 0 & 0 & 0 & 0 & $2 \Delta(t p)$ & 0 & $2 \Delta(t q)$ \\
\hline$\Delta\left(t p_{t}\right)$ & $-2(\Delta t)^{2}$ & 0 & $2\left(\Delta p_{t}\right)^{2}$ & 0 & 0 & 0 & $-\Delta(t q)$ & $\Delta\left(p_{t} p\right)$ & $-\Delta(t p)$ & $\Delta\left(p_{t} q\right)$ \\
\hline$\left(\Delta p_{t}\right)^{2}$ & $-4 \Delta\left(t p_{t}\right)$ & $-2\left(\Delta p_{t}\right)^{2}$ & 0 & 0 & 0 & 0 & $-2 \Delta\left(p_{t} q\right)$ & 0 & $-2 \Delta\left(p_{t} p\right)$ & 0 \\
\hline$(\Delta q)^{2}$ & 0 & 0 & 0 & 0 & $2(\Delta q)^{2}$ & $4 \Delta(q p)$ & 0 & $2 \Delta\left(p_{t} q\right)$ & $2 \Delta(t q)$ & 0 \\
\hline$\Delta(q p)$ & 0 & 0 & 0 & $-2(\Delta q)^{2}$ & 0 & $2(\Delta p)^{2}$ & $-\Delta(t q)$ & $\Delta\left(p_{t} p\right)$ & $\Delta(t p)$ & $-\Delta\left(p_{t} q\right)$ \\
\hline$(\Delta p)^{2}$ & 0 & 0 & 0 & $-4 \Delta(q p)$ & $-2(\Delta p)^{2}$ & 0 & $-2 \Delta(t p)$ & 0 & 0 & $-2 \Delta\left(p_{t} p\right)$ \\
\hline$\Delta(t q)$ & 0 & $\Delta(t q)$ & $2 \Delta\left(p_{t} q\right)$ & 0 & $\Delta(t q)$ & $2 \Delta(t p)$ & 0 & $\Delta\left(t p_{t}\right)$ & $(\Delta t)^{2}$ & $(\Delta q)^{2}$ \\
\hline$\Delta\left(p_{t} p\right)$ & $-2 \Delta(t p)$ & $-\Delta\left(p_{t} p\right)$ & 0 & $-2 \Delta\left(p_{t} q\right)$ & $-\Delta\left(p_{t} p\right)$ & 0 & $-\Delta\left(t p_{t}\right)$ & 0 & $-(\Delta p)^{2}$ & $-\left(\Delta p_{t}\right)^{2}$ \\
& & & & & & & $-\Delta(q p)$ & & $(\Delta p)$ & \\
\hline$\Delta(t p)$ & 0 & $\Delta(t p)$ & $2 \Delta\left(p_{t} p\right)$ & $-2 \Delta(t q)$ & $-\Delta(t p)$ & 0 & $-(\Delta t)^{2}$ & $(\Delta p)^{2}$ & 0 \\
\hline$\Delta\left(p_{t} q\right)$ & $-2 \Delta(t q)$ & $-\Delta\left(p_{t} q\right)$ & 0 & 0 & $\Delta\left(p_{t} q\right)$ & $2 \Delta\left(p_{t} p\right)$ & $-(\Delta q)^{2}$ & $\left(\Delta p_{t}\right)^{2}$ & $\Delta\left(t p_{t}\right)$ & $-\Delta(q p)$ \\
& & & & & & & & $-\Delta\left(t p_{t}\right)$ \\
\hline
\end{tabular}

The Poisson brackets between the expectation values and the moments are zero. In Table 3 we provide the Poisson brackets for the second order moments of quantum variables associated with two canonical pairs $\mathbf{t}, \mathbf{p}_{t} ; \mathbf{q}, \mathbf{p}$.

\section{B Coherent state and effective evolution}

Here we consider the constrained system of Section 4.1 in a specific semiclassical state and compare the evolution of "classical" quantities given by the effective quantum theory to properties of the state. This appendix is included to address the riliability of an effective solution to a constrained quantum system through comparison within a specific example.

Recall the constraint:

$$
\mathbf{C}=\mathbf{p}_{t}^{2}-\mathbf{p}^{2}-\mathbf{q}^{2}-m^{2} \mathbb{1} .
$$

Formally, a positive frequency solution of the constraint described in Section 4.1 reduces the system to one canonical degree of freedom that evolves subject to the Hamiltonian $\mathbf{H}=\left(\mathbf{p}^{2}+\mathbf{q}^{2}+m^{2} \mathbb{1}\right)^{\frac{1}{2}}$. We begin by providing the classical trajectory: a canonical pair $q, p$ subject to the Hamiltonian function $H=\left(p^{2}+q^{2}+m^{2}\right)^{\frac{1}{2}}$ evolves according to the equations of motion:

$$
\begin{aligned}
& \frac{\mathrm{d}}{\mathrm{d} t} q=\{q, H\}=p\left(p^{2}+q^{2}+m^{2}\right)^{-\frac{1}{2}}=p H^{-1} \\
& \frac{\mathrm{d}}{\mathrm{d} t} p=\{p, H\}=-q\left(p^{2}+q^{2}+m^{2}\right)^{-\frac{1}{2}}=q H^{-1}
\end{aligned}
$$


Using the fact that $H$ itself is a constant of motion we differentiate the first equation above with respect to time to obtain the second order equation

$$
\frac{\mathrm{d}^{2}}{\mathrm{~d} t^{2}} q=H^{-1} \frac{\mathrm{d}}{\mathrm{d} t} p=-H^{-2} q
$$

This equation has the general solution

$$
q(t)=A \sin \left(\frac{t}{H}\right)+B \cos \left(\frac{t}{H}\right)
$$

where $A$ and $B$ are constants. It follows that

$$
p(t)=H \frac{\mathrm{d}}{\mathrm{d} t} q=A \cos \left(\frac{t}{H}\right)-B \sin \left(\frac{t}{H}\right)
$$

and thus $H=\sqrt{A^{2}+B^{2}+m^{2}}$. The classical phase-space trajectory is therefore a circle of radius $\sqrt{A^{2}+B^{2}}$ traversed with the angular frequency $\left(A^{2}+B^{2}+m^{2}\right)^{-\frac{1}{2}}$.

Effective equations of motion are set up in much the same way as their classical counterparts. Phase-space degrees of freedom are $q, p,(\Delta q)^{2},(\Delta p)^{2}, \Delta(q p)$. The time evolution is generated by the function $E$ of equation (18) through the quantum Poisson bracket

$$
\begin{aligned}
\frac{\mathrm{d}}{\mathrm{d} t} q & =\frac{p}{\sqrt{p^{2}+q^{2}+m^{2}}}+\frac{p(\Delta q)^{2}\left(2 q^{2}-p^{2}-m^{2}\right)+q \Delta(q p)\left(4 p^{2}-2 q^{2}-2 m^{2}\right)-3 p(\Delta p)^{2}\left(q^{2}+m^{2}\right)}{2\left(p^{2}+q^{2}+m^{2}\right)^{\frac{5}{2}}} \\
\frac{\mathrm{d}}{\mathrm{d} t} p & =\frac{-q}{\sqrt{p^{2}+q^{2}+m^{2}}}+\frac{3 q(\Delta q)^{2}\left(p^{2}+m^{2}\right)-p \Delta(q p)\left(4 q^{2}-2 p^{2}-2 m^{2}\right)-q(\Delta p)^{2}\left(2 p^{2}-q^{2}-m^{2}\right)}{2\left(p^{2}+q^{2}+m^{2}\right)^{\frac{5}{2}}} \\
\frac{\mathrm{d}}{\mathrm{d} t}(\Delta q)^{2} & =\frac{2 \Delta(q p)\left(q^{2}+m^{2}\right)-2(\Delta q)^{2} q p}{\left(p^{2}+q^{2}+m^{2}\right)^{\frac{3}{2}}} \quad, \quad \frac{\mathrm{d}}{\mathrm{d} t}(\Delta p)^{2}=\frac{2(\Delta p)^{2} q p-2 \Delta(q p)\left(q^{2}+m^{2}\right)}{\left(p^{2}+q^{2}+m^{2}\right)^{\frac{3}{2}}} \\
\frac{\mathrm{d}}{\mathrm{d} t} \Delta(q p) & =\frac{(\Delta p)^{2}\left(q^{2}+m^{2}\right)-(\Delta q)^{2}\left(p^{2}+m^{2}\right)}{\left(p^{2}+q^{2}+m^{2}\right)^{\frac{3}{2}}} .
\end{aligned}
$$

The first term in each of the first two equations is identical to the classical equations of motion, the extra terms constitute the leading order quantum corrections. The system of equations is straightforward to evolve numerically for a sufficiently short time starting from a state that is initially semiclassical.

The quantum evolution of positive frequency solutions is governed by the Schrödinger equation

$$
-\frac{\hbar}{i} \frac{\mathrm{d}}{\mathrm{d} t} \Psi(x, t)=\left(\mathbf{p}^{2}+\mathbf{q}^{2}+m^{2} \mathbb{1}\right)^{\frac{1}{2}} \Psi(x, t) .
$$

The square root operator has the same eigenstates $\left\{\varphi_{n}(x)\right\}_{n=0, \ldots \infty}$; as the one dimensional quantum harmonic oscillator, with eigenvalues $\lambda_{n}=\sqrt{2\left(n+\frac{1}{2}\right) \hbar+m^{2}}$. A wavefunction can be evolved by decomposing it into the eigenstates of the Hamiltonian. If the state at 
$t=0$ is given by $\Psi(x, 0)=\sum_{n=0}^{\infty} c_{n} \varphi_{n}(x)$, where $c_{n}$ are constant complex numbers, then at any other time, the wavefunction is

$$
\Psi(x, t)=\sum_{n=0}^{\infty} c_{n} \exp \left(-i \frac{\lambda_{n} t}{\hbar}\right) \varphi_{n}(x) .
$$

To compute the expectation values we write $\mathbf{q}=\sqrt{\hbar / 2}\left(\hat{a}^{*}+\hat{a}\right), \mathbf{p}=i \sqrt{\hbar / 2}\left(\hat{a}^{*}-\hat{a}\right)$, where $\hat{a}^{*}$ and $\hat{a}$ are the usual creation and annihilation operators of the quantum harmonic oscillator. One finds

$$
\begin{aligned}
& \langle\Psi, \mathbf{q} \Psi\rangle(t)=\sum_{n=0}^{\infty} \sqrt{2 \hbar(n+1)} \operatorname{Re}\left[\bar{c}_{n} c_{n+1} \exp \left(-i t \frac{\lambda_{n+1}-\lambda_{n}}{\hbar}\right)\right] \\
& \langle\Psi, \mathbf{p} \Psi\rangle(t)=\sum_{n=0}^{\infty} \sqrt{2 \hbar(n+1)} \operatorname{Im}\left[\bar{c}_{n} c_{n+1} \exp \left(-i t \frac{\lambda_{n+1}-\lambda_{n}}{\hbar}\right)\right]
\end{aligned}
$$

In a similar manner one can obtain expressions for the moments of $\mathbf{q}$ and $\mathbf{p}$.

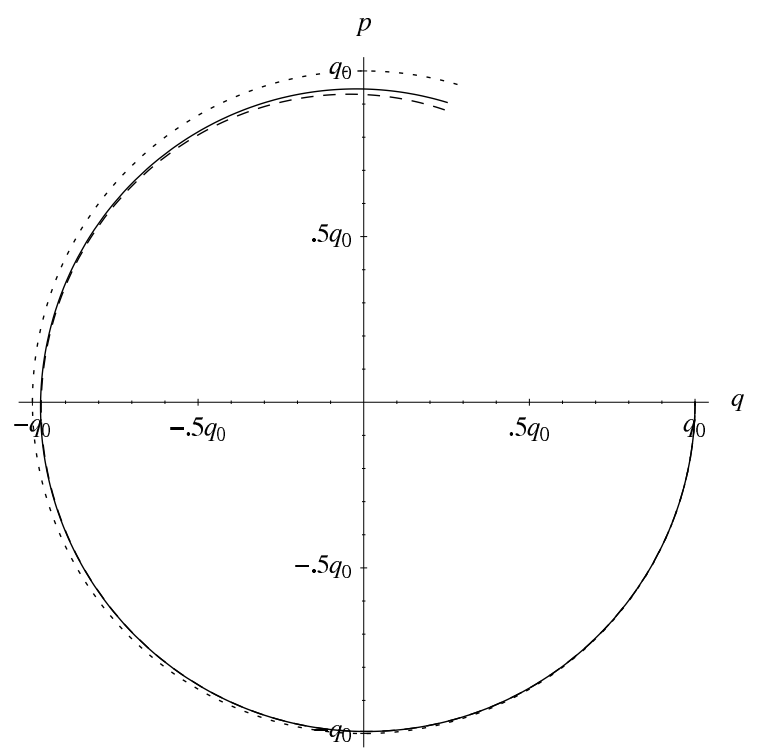

Figure 1: Classical (dotted), coherent state (solid) and effective (dashed) phase space trajectories, evolved for $0 \leq t \leq 5 q_{0}$.

In order to complete the comparison we select a semiclassical state with a known decomposition into the eigenstates $\left\{\varphi_{n}(x)\right\}$. A simple choice is to set the initial wavefunction to be a coherent state of the harmonic oscillator

$$
c_{n}=\exp \left(-\frac{|\alpha|^{2}}{2}\right) \frac{\alpha^{n}}{\sqrt{n !}}, \quad \alpha \in \mathbb{C}
$$

which we can consider as a kinematical coherent state for our system. For the nonrelativistic harmonic oscillator, as time goes on $\alpha$ changes, but the shape of the state 
is preserved. Clearly, this is not the case for our relativistic evolution: Combining (22) with (21), we have an evolving state which, expanded in harmonic oscillator stationary states, has coefficients

$$
c_{n} e^{-i \lambda_{n} t / \hbar}=\frac{1}{\sqrt{n !}} e^{-|\alpha|^{2} / 2} \alpha^{n} e^{-i \sqrt{2 n+1+m^{2} / \hbar^{2}} t} .
$$

Due to the square root in this relativistic model, these coefficients are not of the coherent state form (22) unless $t \neq 0$. The physical states we obtain are not dynamical coherent states; quantum back-reaction ensues which in the effective treatment is captured by the coupling terms between moments and expectation values in (18).

For a specific numerical example, we set $\alpha=\frac{q_{0}}{\sqrt{2 \hbar}}$ so that at $t=0$ the state is a Gaussian peaked about $q=q_{0}$ and $p=0$, with zero covariance and minimal spread

$$
(\Delta q)^{2}=(\Delta p)^{2}=\frac{\hbar}{2}, \quad \Delta(q p)=0 .
$$

We take these as initial values for the numerical evolution of the effective equations for the system. We assume the two physical scales to be separated by a single order of magnitude by setting $\frac{q_{0}}{\sqrt{\hbar}}=10$. For simplicity we set $m=0$. Depicted in FIG. 1 are the classical, coherent and effective quantum phase-space trajectories starting from the same initial state. One can see that the effective equations describe the correct semiclassical trajectory for much of the evolution displayed. An internal measure of consistency is the size of second order moments. From FIG. 2, we see that the semiclassical approximation clearly breaks down after approximately $t=2 q_{0}$, as $(\Delta p)^{2}$ becomes too large. The same figure demonstrates that up until that time the evolution of the moments themselves is very well approximated by the effective equations. The other two moments display similar behavior.

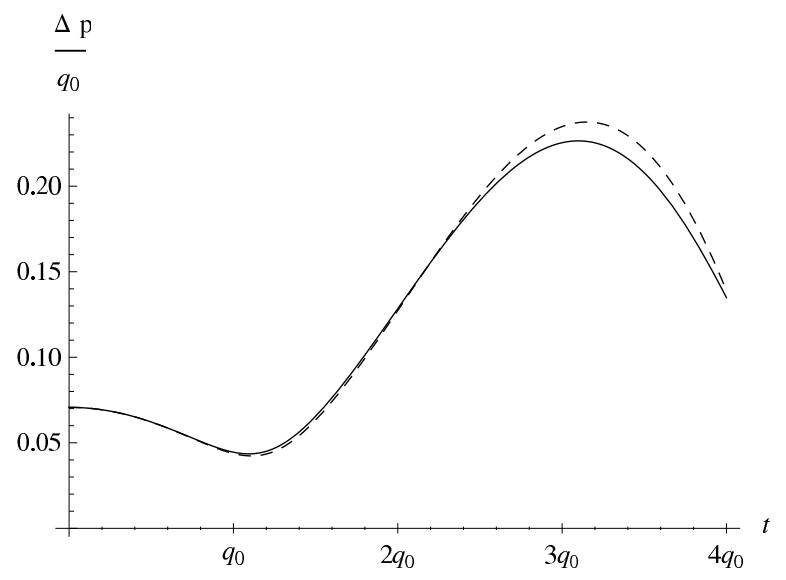

Figure 2: Coherent state (solid) and effective (dashed) evolution of the second order moment $\Delta p=\sqrt{(\Delta p)^{2}}$ in units of $q_{0}$. 


\section{References}

[1] M. Bojowald and A. Skirzewski, Effective Equations of Motion for Quantum Systems, Rev. Math. Phys. 18 (2006) 713-745, math-ph/0511043

[2] M. Bojowald, B. Sandhöfer, A. Skirzewski, and A. Tsobanjan, Effective constraints for quantum systems, Rev. Math. Phys. 21 (2009) 111-154, arXiv:0804.3365.

[3] H. Feshbach and F. Villars, Elementary Relativistic Wave Mechanics of Spin 0 and Spin 1/2 Particles, Rev. Mod. Phys. 30 (1958) 24-45

[4] J. B. Hartle and D. Marolf, Comparing Formulations of Generalized Quantum Mechanics for Reparametrization-Invariant Systems, Phys. Rev. D 56 (1997) 6247-6257, gr-qc/9703021

[5] M. Bojowald, Large scale effective theory for cosmological bounces, Phys. Rev. D 75 (2007) 081301(R), gr-qc/0608100

[6] M. Bojowald, Dynamical coherent states and physical solutions of quantum cosmological bounces, Phys. Rev. D 75 (2007) 123512, gr-qc/0703144

[7] M. Bojowald, Harmonic cosmology: How much can we know about a universe before the big bang?, Proc. Roy. Soc. A 464 (2008) 2135-2150, arXiv:0710.4919.

[8] M. Bojowald and R. Tavakol, Recollapsing quantum cosmologies and the question of entropy, Phys. Rev. D 78 (2008) 023515, arXiv:0803.4484]

[9] W. F. Blyth and C. J. Isham, Quantization of a Friedmann universe filled with a scalar field, Phys. Rev. D 11 (1975) 768-778

[10] A. Ashtekar, T. Pawlowski, and P. Singh, Quantum Nature of the Big Bang: An Analytical and Numerical Investigation, Phys. Rev. D 73 (2006) 124038, [gr-qc/0604013

[11] M. Bojowald, H. Hernández, and A. Skirzewski, Effective equations for isotropic quantum cosmology including matter, Phys. Rev. D 76 (2007) 063511, arXiv:0706.1057]

[12] M. Bojowald, Quantum nature of cosmological bounces, Gen. Rel. Grav. 40 (2008) 2659-2683, arXiv:0801.4001]

[13] M. Bojowald, How quantum is the big bang?, Phys. Rev. Lett. 100 (2008) 221301, arXiv:0805.1192

[14] M. Bojowald, What happened before the big bang?, Nature Physics 3 (2007) 523-525

[15] M. Bojowald, Comment on "Quantum bounce and cosmic recall", Phys. Rev. Lett. 101 (2008) 209001, arXiv:0811.2790 
[16] M. Bojowald and T. Strobl, Poisson Geometry in Constrained Systems, Rev. Math. Phys. 15 (2003) 663-703, hep-th/0112074]

[17] P. A. M. Dirac, Lectures on Quantum Mechanics, Yeshiva Press, 1969

[18] R. Haag, Local Quantum Physics, Springer-Verlag, Berlin, Heidelberg, New York, 1992

[19] M. Varadarajan, On the resolution of the big bang singularity in isotropic Loop Quantum Cosmology, Class. Quantum Grav. 26 (2009) 085006, arXiv:0812.0272]

[20] T. Cailleteau, A. Cardoso, K. Vandersloot, and D. Wands, Singularities in loop quantum cosmology, Phys. Rev. Lett. 101 (2008) 251302, arXiv:0808.0190] 